\title{
Shear viscosity of the Lennard-Jones chain fluid in its gaseous, supercritical, and liquid states
}

\author{
Guillaume Galliero* and Christian Boned \\ Laboratoire des Fluides Complexes (UMR-5150 with CNRS and TOTAL), Université de Pau et des Pays de l'Adour, BP 1155, \\ F-64013 PAU Cedex, France
}

(Received 6 November 2008; published 4 February 2009)

\begin{abstract}
Extensive nonequilibrium molecular dynamics (NEMD) simulations have been carried out in order to estimate the Newtonian shear viscosity of a fluid composed of short chains (up to 16 segments) of jointed spheres [Lennard-Jones chain (LJC)] over a large range of thermodynamic conditions. Using the NEMD results, it is shown that the zero-density contribution decreases with the chain length for a given temperature and is simply proportional to $N^{-1 / 2}$, where $N$ is the number of spheres composing the chain. In addition, it has been noticed that the residual shear viscosity is proportional to the chain length. Then, using these results, a relation is proposed to correlate the shear viscosity of the LJC fluid using the LJ fluid (the monomer) as a reference. It is shown that this correlation is able to provide an excellent estimation of the LJC fluid viscosity compared to NEMD results for $N \leqslant 16$ over the domain $0 \leqslant \rho^{*} \leqslant 1.1$ and $0.7 \leqslant T^{*} \leqslant 6$. Finally, it is shown that the LJC model is unambiguously more efficient than a simple LJ approximation when applied to estimate the shear viscosity of $n$-butane, if only the sphere or segment diameter is used as an adjustable parameter in both models.
\end{abstract}

DOI: 10.1103/PhysRevE.79.021201

PACS number(s): 66.20.Cy, 02.70.Ns

\section{INTRODUCTION}

For simple fluids, kinetic theories [1] are able to provide accurate transport properties in the low-density limit-in particular, shear viscosity-but the situation is more complex when dealing with dense fluids. In fact, in such conditions where strong velocity correlation exists [2], no rigorous theory is yet available for an exact estimation of the viscosity in terms of interaction potentials.

One possible way to improve the molecular based theories of viscosity is the use of molecular dynamics (MD) simulations [3] on model fluid (interaction potential + molecular description). These numerical simulations can provide "exact" results for a given fluid model and so can serve both as a direct test of the existing theories and as a way to construct new theories and correlations. Such an approach has been widely employed in order to develop molecular-based equations of state $[2,4]$, like the statistical association fluid theory (SAFT) ones [5]. Nevertheless, less effort has been devoted to transport properties, especially those that are a collective property (viscosity, thermal conductivity).

However, in the recent years, improvements toward the modelling of the viscosity of simple spherical fluids model have been achieved using MD simulations. Among them is the hard sphere, sometimes called rigid-sphere, fluid [6]. This fluid model is probably the simplest model and is rather easy to handle and so is often used as basis (a reference) to construct theory as done for thermodynamic properties. Based on Enskog's solution of the Boltzmann equation [1], rather good results can be obtained in some circumstances when applied to real fluids [7]. However, this oversimplified fluid model is not adapted for long-chain molecules or for asymmetric mixtures $[7,8]$. It should be noted that, very recently, extensions to a model describing the viscosity of

\footnotetext{
*Corresponding author. FAX: +33 559407695 . guillaume.galliero@univ-pau.fr
}

hard-sphere chains have been proposed $[8,9]$ which may take benefit from recent findings concerning the maximally random jammed state of tangent hard spheres [10] An alternative to the hard-sphere reference is to use a more realistic potential as a basis, including directly dispersive forces, such as the Lennard-Jones (LJ) potential. Various approaches have been proposed in order to predict the viscosity of a fluid composed of spheres interacting through a LJ potential (LJ fluid), some having a more or less theoretical basis [11-15], some being purely empirical correlations [16-18]. A reasonable prediction of the viscosity of nonassociative real fluids can be reached using this fluid model [18-21], but because of the spherical approximation, such an approach is less adequate for nonspherical molecules as well as for asymmetric mixtures $[18,20]$. Another weakness of the LJ model comes from the fact that different molecular parameters set should often be used for estimating different properties [22,23], which clearly emphasizes the limit of such a spherical approximation.

In this work, it is proposed to perform extensive nonequilibrium MD (NEMD) simulations [24] on the LJ chain (LJC) fluid model-i.e., a molecule described by chains of jointed spheres interacting through a LJ potential (for nonbonded interactions). The aim is both to provide an accurate database of the LJC shear viscosity and to develop a "chain term" for the LJC viscosity, as already done for the LJC equation of state [25]. This chain term, combined with an accurate LJ reference term, is aimed at providing a good estimation of the shear viscosity of the LJC fluid model for short chains (i.e., 16 spheres at most) and a very large range of thermodynamic conditions covering gas, liquid, and supercritical conditions. It should be mentioned that a similar approach has been already attempted for the self-diffusion coefficient of the LJC fluid, a transport property that is easier and quicker to obtain using MD simulations than the shear viscosity [26-28]. In addition, from the rheological point of view, recent works exist in the literature that deal with the Weeks-Chandler-Andersen (purely repulsive LJ sphere) + FENE (bonded interaction) fluid model [29], which is to a 
certain extent similar to the LJC fluid. It is worth to mention as well the elastic LJ model introduced in Ref. [30], which exhibits gelation and filamentous networks.

In Sec. II, the LJC fluid model employed in this work is described, followed by a brief description of the way shear viscosity is computed using NEMD simulations with corresponding numerical details. Section III starts by a brief analysis of possible non-Newtonian effects and the spring stiffness influence. Then, LJC shear viscosity results for both low- and high-density conditions are provided, analyzed, and discussed. In addition, the proposed LJC viscosity correlation is applied to normal butane. Finally, Sec. IV provides a summary of the obtained results.

\section{MODEL AND THEORY}

\section{A. Fluid model}

In this work, we consider a LJC model (a multibead model with strong harmonic bond and excluded volume [31]), where each LJC molecule is modeled as a set of $N$ jointed segments (spheres). The intermolecular and nonbonded intramolecular interactions (corresponding to nonadjacent segments) are described by the truncated LJ 12-6 potential

$$
U_{L J}= \begin{cases}4 \varepsilon\left[\left(\frac{\sigma}{r}\right)^{12}-\left(\frac{\sigma}{r}\right)^{6}\right] & \text { if } r<r_{c}, \\ 0 & \text { if } r \geqslant r_{c},\end{cases}
$$

where $\varepsilon$ is the potential strength, $\sigma$, the "atomic diameter," which is the distance at which the potential is null, $r$ the center-to-center distance between the two segments considered, and $r_{c}$ the cutoff radius $(=2.5 \sigma$ in this work). The bonded intramolecular interactions are modeled by a simple spring-type potential with an equilibrium distance equal to $\sigma$ :

$$
U_{\text {bond }}=-\frac{1}{2} k(r-\sigma)^{2},
$$

where $k$ is the spring constant usually taken equal to $3000 \varepsilon / \sigma^{2}$ (as in the paper of Johnson et al. [25]). However, the influence of $k$ on the shear viscosity has been considered in this article.

In the following, the variables are expressed in dimensionless units, noted with a star as superscript, using the monomer parameters $(\sigma, \varepsilon$, and $M$, where $M$ is the mass of the monomer-i.e., $M=M_{m o l} / N$, where $M_{m o l}$ is the molecular weight) as characteristic length, energy, and mass. Concerning the temperature $T$, density $\rho$, pressure $P$, and shear viscosity $\eta$, the corresponding dimensionless variables are

$$
T^{*}=\frac{k_{B} T}{\varepsilon}, \quad \rho^{*}=\frac{N_{T} \sigma^{3}}{V}, \quad P^{*}=\frac{P \sigma^{3}}{\varepsilon}, \quad \eta^{*}=\eta \frac{\sigma^{2}}{\sqrt{M \varepsilon}},
$$

where $k_{B}$ is the Boltzmann constant, $N_{T}$ the total number of spheres, and $V$ the volume of the simulation box.

\section{B. Nonequilibrium molecular dynamics}

The procedure employed to estimate shear viscosity from MD simulations is based on a boundary driven nonequilib- rium algorithm proposed in Ref. [24], which is compatible with periodic boundary conditions.

In this approach, the simulation box is divided into $N_{s}$ slabs (=24 in this work) along the $z$ direction. Then, the fluid is sheared using a net exchange of the momentum along the $x$ direction, which is performed between the central part of the simulation box, $N_{s} / 2$ and $N_{s} / 2+1$, and the edge layers, slab 1 and $N_{s}$. To do so, we select the two spheres in slabs 1 and $N_{s}$ with the largest negative $x$ components of the momentum and the two spheres in slabs $N_{s} / 2$ and $N_{s} / 2+1$ with the largest positive $x$ components of the momentum. Then, the $x$ components of the velocity (in pure fluids) $v_{x}{ }^{*}$ between the spheres involved are exchanged. This procedure keeps constant the overall energy and momentum and corresponds to a redistribution of a certain amount of momentum, $\Delta p_{x}{ }^{*}$, in the simulation box [24]. This exchange is done every $A$ time steps (exchange frequency, usually $\sim 100$ [32]) and so corresponds to a transverse linear momentum flux (shear stress) $J_{x z}^{*}$ of

$$
J_{x z}^{*}=\frac{\Delta p_{x}^{*}}{2 A \delta t^{*} L_{x}^{*} L_{y}^{*}},
$$

where $\delta t^{*}$ is the time step and $L_{x}{ }^{*}$ and $L_{y}{ }^{*}$ are the lengths of the simulation box, respectively, in the $x$ and $y$ directions. It should be noted that when $A$ is small, which corresponds to a large applied shear, non-Newtonian behavior may appear. This effect will be studied in the following.

At the stationary state, the system exhibits a biperiodical velocity profile and, in the linear response regime, the shear rate $\dot{\gamma}^{*}=\Delta v_{x}^{*} / \Delta z^{*}$ is evaluated thanks to the local velocity computed in each slabs. Then, the viscosity of the system is simply deduced from Eq. (4) and Newton's law [32]:

$$
J_{x z}^{*}=-\eta^{*} \frac{\Delta v_{x}^{*}}{\Delta z^{*}} \text {. }
$$

The slabs where the exchanges are performed, as well as their first neighbors, have been discarded to measure the shear rate.

\section{Simulation details}

We have used a homemade code already validated for various thermophysical properties $[18,33]$. The system was set up by placing $N_{\text {mol }}$ molecules in a cubic simulation box, where $N_{m o l}$ was fixed to 750 when $N=2,375$ when $N=4,300$ when $N=8$, and 250 when $N=16$. Classical periodic boundary conditions combined with a Verlet neighbors list have been applied [34]. A reduced time step $\delta t^{*}$ equal to 0.003 has been employed. To integrate the equation of motion, the velocity Verlet algorithm is used [3]. To maintain the desired temperature during simulations, a Berendsen thermostat [35] with a large time constant equal to $1000 \delta t^{*}$ has been utilized to avoid disturbances [36]. After equilibration, each run has been performed during $(0.5-2) \times 10^{7}$ nonequilibrium time steps for the computation of viscosity. The CPU time per particle and time step is of the order of $10^{-5} \mathrm{~s}$ on AMD Opteron $2.4 \mathrm{GHz}$.

For the computation of the internal energy and the pressure, during equilibrium runs, the usual long-range correc- 


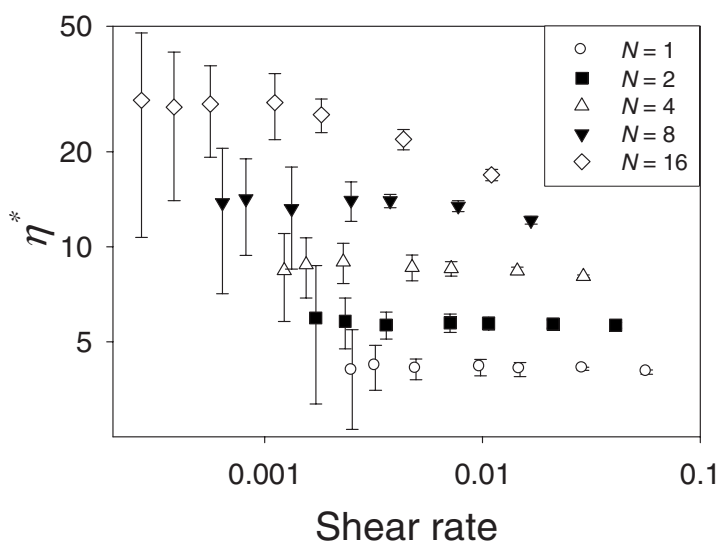

FIG. 1. Influence of the shear rate $\dot{\gamma}^{*}$ on the apparent shear viscosity for different LJC fluids at $T^{*}=1$ and $\rho^{*}=0.9$.

tions have been included [34]. In order to estimate errors on the variables computed, the sub-block average method has been applied [3].

\section{RESULTS}

\section{A. Preliminary results}

In a first step, we have compared our equilibrium simulations results with those of Johnson et al. [25] for both pressure and internal energy. Simulations on stable fluid states have been performed for $T^{*}=2,3$, and $4, \rho^{*}=0.8,0.9$, and 1 , and $N=2,4,8$, and 16 . For these dense stable conditions (27 points), our results are in excellent agreement with those of Johnson et al. [25]. More precisely, we have obtained average absolute deviation (AAD) of $0.38 \%$ in pressure and of $0.10 \%$ in internal energy relatively to the results of Johnson et al. [24].

\section{Non-Newtonian effects}

Because of the huge shear rates accessible by NEMD simulations, non-Newtonian behavior, such as shear thinning, may be noted for simple fluids as the LJ one $[11,31,37]$. In order to ensure that our simulations allow us to obtain the Newtonian shear viscosity, the influence of the shear rate on the apparent viscosity is analyzed for a dense liquid state at $T^{*}=1$ and $\rho^{*}=0.9$ and for $N=1,2,4,8$, and 16 . To do so, simulations have been performed for different exchange frequencies $A$ (see Sec. II B), by taking values equal to 25 (large shear), 50, 100, 150, 300, 450, and 600 (small shear).

The results shown in Fig. 1 indicate that shear thinning, as expected [38], appears for the longest chains and for the largest shear rate. In addition, for all chain lengths the apparent viscosity reaches a plateau corresponding to the Newtonian regime when the shear rate is decreased. The plateau is reached for a shear-rate value that decreases when the chain length increases: e.g., $\dot{\gamma}^{*} \approx 0.1$ for $N=1$ [38] and $\dot{\gamma}^{*}$ $\approx 0.001$ for $N=16$ (this work).

In the following computations, we have employed exchange frequencies varying from 150 to 300 , which correspond to $J_{x z}{ }^{*} \approx 0.02-0.04$. These values allow us to limit the uncertainties (which increase when $\dot{\gamma}^{*}$ decreases because of

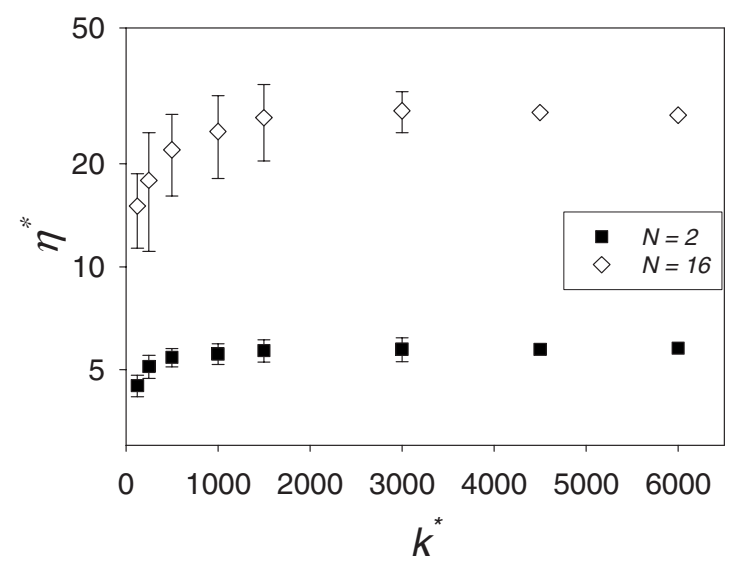

FIG. 2. Influence of the spring constant $k^{*}$ on the viscosity for two different LJC fluids $(N=2$ and 16$)$ at $T^{*}=1$ and $\rho^{*}=0.9$.

the thermal noise) and ensure to stay in the Newtonian regime.

\section{Spring stiffness influence}

Another parameter that may influence the viscosity measured is the way the bonds are dealt with. Therefore, we have evaluated the impact of the amplitude of the spring constant, $k^{*}$ in Eq. (2), on the viscosity obtained. Thus, for the same state than in the previous section-i.e., $T^{*}=1$ and $\rho^{*}=0.9$-we have computed the shear viscosity for $k^{*}=125$, $250,500,1000,1500,3000,4500$, and 6000 for both $N=2$ and $N=16$. It has been verified that the reduced time step used, $\delta t^{*}=0.003$, was small compared to $2 \pi / \sqrt{k^{*}}$ and that no energy drift was noted.

Figure 2 shows that the value of the spring constant affects the viscosity computed, but only for the lowest values of $k^{*}$. In that case, this decrease of $\eta^{*}$ when $k^{*}$ decreases is consistent with the fact that for very weak $k^{*}$, the shear viscosity of the LJC fluid should tend to that of the monomer $\left(\eta_{L J}^{*} \approx 4.0\right.$ [18]). Elsewhere, $\eta^{*}$ becomes independent of $k^{*}$, for the case studied here, when $k^{*}$ is above $\sim 1000$. Consequently, for the value used in the following, $k^{*}=3000$, the large $k^{*}$ limit is attained which ensures that the fluids studied are effectively composed of jointed chains with a bond length of $\sigma$. For viscosity estimation, the choice of a continuous harmonic potential to describe covalent bond instead of a constrained (rigid) approach [3] is merely for computational convenience, especially concerning the NEMD algorithm used [32].

\section{B. Low-density contribution}

Usually the viscosity is described as the sum of three terms [39]:

$$
\eta=\eta_{0}+\eta_{r}+\eta_{c p}
$$

where $\eta_{0}$ corresponds to the "zero-density" contribution, $\eta_{c p}$ the viscosity enhancement appearing close to the critical point, and $\eta_{r}$ the residual viscosity. In this work, we will neglect the $\eta_{\mathrm{cp}}$ contribution which is important only in the vicinity of the critical point. 
The zero-density contribution can be estimated for the LJ fluid (monomer) using the Chapman-Enskog solution of the Boltzmann equation $[1,40]$ :

$$
\eta_{0}^{*}=\frac{5}{16 \Omega_{v}} \sqrt{\frac{T^{*}}{\pi}}
$$

where $\Omega_{v}$ is the collision integral that can be estimated using the correlation provided by Neufeld et al. [41]. Unfortunately its extension to polyatomic molecules is difficult to achieve [1] and, to the best of our knowledge, such a relation does not exist for the LJC fluid model. In addition, MD simulations can be performed only at nonzero density and consequently the zero-density contribution can only be obtained by an extrapolation of the results to zero density. Moreover, it seems that NEMD simulations for too low densities suffer from finite-size effects as already noticed in Ref. [42].

To circumvent these difficulties, we propose a simple approach to deduce the zero-density viscosity of the LJC fluid using NEMD simulations on non-zero-density systems. To do so, we start from the fact that the transfer of linear momentum $J_{x z}^{*}$ is induced by the particle displacement (translation) and by interactions between particles (collision). In this frame, the corresponding microscopic formulation of the linear momentum flux is [3]

$$
J_{x z}^{*}=-\frac{1}{V^{*}}\left(\sum_{i=1}^{N} v_{i, x}^{*} v_{i, z}^{*}+\sum_{i=1}^{N-1} \sum_{j>i}^{N} r_{i j, x}^{*} F_{i j, z}^{*}\right),
$$

where $v_{i, x}^{*}$ the $x$ component of the velocity of particle $i, r_{i j, x}^{*}$ the $x$ component of the distance vector between particle $i$ and $j$, and $F_{i j, z}^{*}$ the $z$ component of the force of particle $i$ acting on particle $j$. The first term on the right-hand side of Eq. (8) represents the translational contribution and the second term the collisional contribution to the momentum flux. Thus, using this decomposition and Eq. (5), it is possible to consider that the shear viscosity measured during NEMD simulations is composed of two terms:

$$
\eta^{*}=\eta_{t}^{*}+\eta_{c}^{*}
$$

where $\eta_{t}^{*}$ is a translational viscosity (sometimes called kinetic viscosity [38]) and $\eta_{c}{ }^{*}$ a collisional viscosity (sometimes called potential viscosity [38]). It should be noted that such a decomposition is similar to the one used in Ref. [38], or in Ref. [43] for energy transport. However, it is worth to notice that this decomposition is different from the one employed during equilibrium molecular simulations (based on the Green-Kubo relation), which induces a cross-product (a third contribution) between translation and collision [44].

By definition, the translational viscosity $\eta_{t}{ }^{*}$ tends to the zero-density viscosity $\eta_{0}{ }^{*}$ when $\rho^{*}$ tends to zero. However, the link between $\eta_{0}^{*}$ and the value of $\eta_{t}^{*}$ for the non-zerodensity condition is not obvious. Therefore, for different stable thermodynamic conditions of the LJ fluid, at $T^{*}=1,2$, 4 , and 6 and $\rho^{*}=0.2,0.4,0.6$, and 0.8 , we have estimated $\eta_{t}{ }^{*}$ during NEMD simulations and compared them with $\eta_{0}{ }^{*}$ calculated using Eq. (7).

Results provided in Fig. 3 indicate that $\eta_{t}{ }^{*} / \eta_{0}{ }^{*}$ tends to

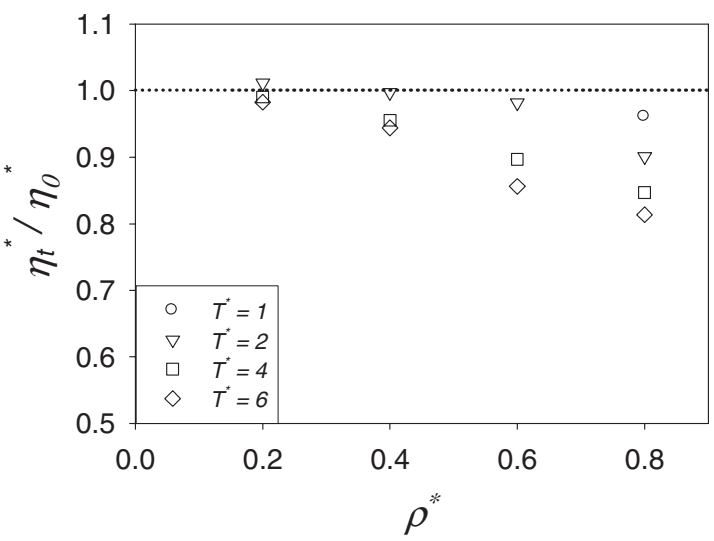

FIG. 3. Ratio between the translational viscosity computed during NEMD simulations and the zero-density viscosity, Eq. (7), of a $\mathrm{LJ}$ fluid for various thermodynamic states.

slowly decrease with density and temperature. This trend is consistent with the findings of Meier et al. [44]. A very interesting feature that emerges from the results shown in Fig. 3 is that for $\rho^{*}=0.2$-i.e., at low-density conditions $-\eta_{t}^{*}$ is already approximately equal to $\eta_{0}{ }^{*}$, the relative difference between these quantities being smaller than $2 \%$ whatever the temperature. Thus, when dealing with the LJ fluid $(N=1)$, the evaluation of $\eta_{t}^{*}$ at $\rho^{*}=0.2$ during NEMD simulations provides a very good approximation of $\eta_{0}{ }^{*}$-i.e., $\eta_{0}{ }^{*}\left(T^{*}\right)$ $\approx \eta_{t}^{*}\left(\rho^{*}=0.2, T^{*}\right)$. Assuming that such behavior remains valid not only for $N=1$, but for all $N$, we have computed $\eta_{t}{ }^{*}$ at $\rho^{*}=0.2$ for various $N$ and $T^{*}$ in order to estimate the zero-density viscosity of the LJC fluid.

As can be seen from Fig. $4, \eta_{0}{ }^{*}$ (deduced from $\eta_{t}^{*}$ at $\left.\rho^{*}=0.2\right)$ tends to increase with temperature, whatever $N$, as is usually the case for the viscosity of low-density gases of simple fluids. Moreover, the zero-density viscosity decreases when the chain length increases for a given temperature. This is consistent with the behavior of the viscosity of low-density normal alkanes, which decreases with the chain length for a given temperature.

Another very interesting feature of the results obtained is that $\eta_{0}^{*}$ increases linearly with $N^{-1 / 2}$ for a given temperature; see Fig. 5. This behavior allows us to deduce a relation to

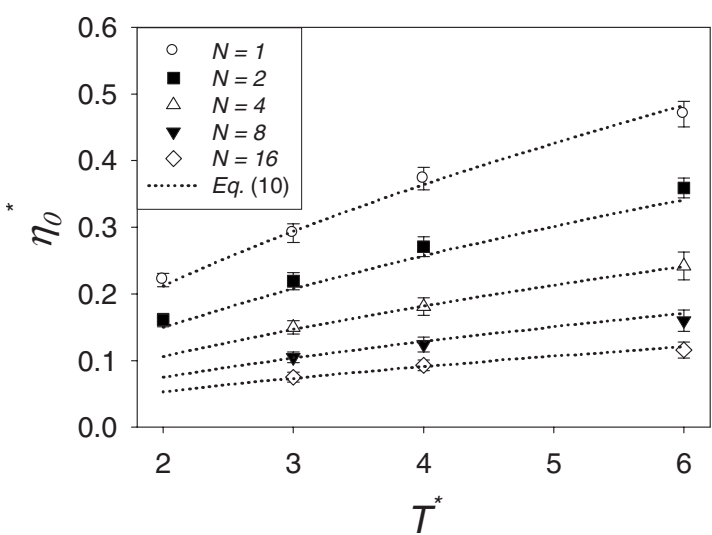

FIG. 4. Zero-density viscosity of the LJC fluids deduced from NEMD simulations. 


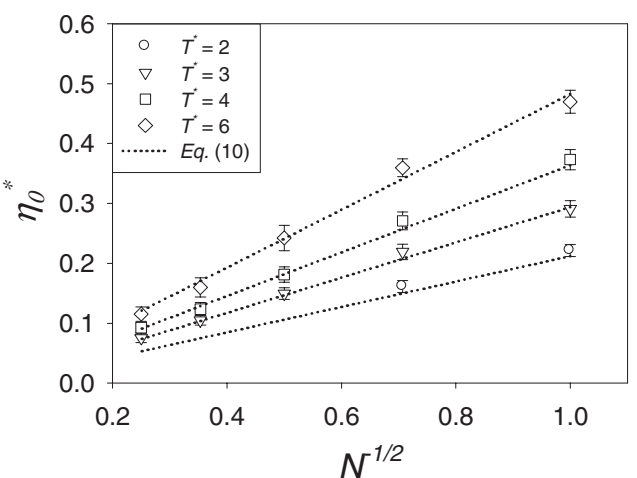

FIG. 5. Zero-density viscosity versus the chain length for various temperatures.

estimate the zero-density of the LJC fluid, which is simply

$$
\eta_{0, L J C}^{*}=\frac{\eta_{0, L J}^{*}}{\sqrt{N}}=\frac{5}{16 \Omega_{v}} \sqrt{\frac{T^{*}}{N \pi}} .
$$

It is worth noting that Eq. (10) ensures that $\eta_{0, L J C}^{*}(N=1)$ $=\eta_{0, L v}^{*}$

As shown in Figs. 4 and 5, Eq. (10) is able to provide an excellent estimation of the zero-density viscosity of the LJC fluid, whatever $N$. More precisely, the AAD between results provided by Eq. (10) and the ones obtained by NEMD simulations is equal to $3.3 \%$ with a maximum deviation $(\mathrm{MxD})$ equal to $7.1 \%$ (for $T^{*}=2$ and $N=2$ ). It is also worth noting that Eq. (10) implies that, in real units, the zero density of a LJC fluid is proportional to $M_{m o l}^{-1 / 2}$ for a given temperature and density.

In fact, Eq. (10) can be supported by a simple analysis of the hard-sphere-chain (HSC) case [i.e., $\Omega_{v}=1$ in Eq. (7)]. In a homonuclear freely jointed chain of hard spheres (composed of segments of diameter $\sigma_{H S C}$ and of mass $M_{H S C}$ ) the mean square of the end-to-end vector $\mathbf{R}$ is such that $\left\langle\mathbf{R}^{2}\right\rangle$ $=N \sigma_{H S C}^{2}$. Assuming that the zero density viscosity of a HSC fluid can be approximated by an equivalent single-hardsphere (HS) model with a diameter $\sigma_{H S}$, we get $\sigma_{H S}^{2}$ $\propto N \sigma_{H S C}^{2}$. So, as $M_{\mathrm{HS}}=N M_{H S C}$, the usual viscosity scaling [cf. Eq. (3)] leads to

$$
\eta_{0, H S}^{*} \propto N^{1 / 2} \eta_{0, H S C}^{*},
$$

which is consistent with what we have noticed using NEMD simulations on the LJC fluid; see Fig. 5.

\section{Residual contribution}

When dealing with short chains, such as those studied in this work $(N \leqslant 16)$, it is expected that the behavior of the LJC viscosity in a dense state is similar to that exhibited by the Rouse model [45] - i.e., a Newtonian viscosity which is proportional to $N$. The transition to the reptation regime, where the viscosity scales as $N^{3.5}$, occurs for chains longer than $N \approx 100$ [46], which are by far longer chains than those studied in this work $(N \leqslant 16)$.

To quantify precisely the non-zero-density Newtonian viscosity of the LJC fluid, we have performed extensive NEMD

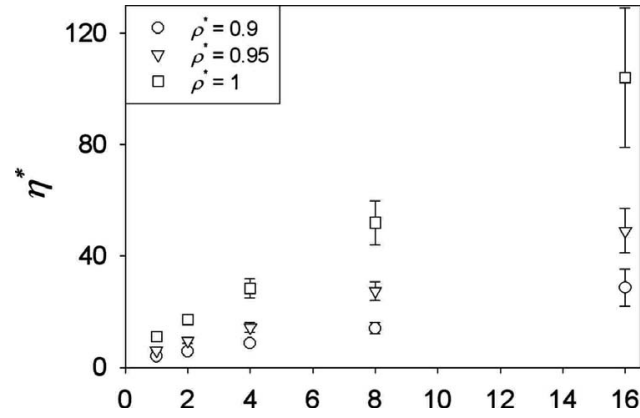

FIG. 6. LJC viscosity versus chain length in the liquid phase at $T^{*}=1$ for three different dense states.

simulations (203 points) for $N=2,4,8$, and 16. Simulations have been performed for a large range of stable thermodynamic conditions covering from $\rho^{*}=0.2$ to $\rho^{*}=1.1$ and from $T^{*}=0.7$ to 6 . All results are provided in the Appendix. Values for $N=1$ have been taken from one of our previous works [18], except for some thermodynamic conditions (not already simulated in Ref. [18]) for which new computations have been performed.

As expected from the Rouse model, Fig. 6 shows that, in dense liquid states (where $\eta_{0}{ }^{*}$ is negligible), the viscosity of the LJC fluid computed by NEMD simulations is proportional to the chain length. In addition, for all situations, the residual viscosity $\left(\eta_{r}{ }^{*}=\eta^{*}-\eta_{0}{ }^{*}\right)$ increases with density, as shown in Fig. 7. A very interesting feature of these NEMD results is that, relatively to the residual viscosity of the monomer (LJ), the residual viscosity of the LJC fluid seems to be weakly dependent on the temperature, whatever the state; see Fig. 7.

Using all these previous results, we have developed an empirical relation (chain term) to express the residual viscosity of the LJC fluid in function of the residual viscosity of the monomer (LJ):

$$
\eta_{r, L J C}^{*}=\eta_{r, L J}^{*}\left[1+(N-1) \frac{\left(\rho^{*}\right)^{c_{1}}}{c_{2}}\right],
$$

where $c_{1}$ and $c_{2}$ are two constants adjusted on the NEMD simulation data, which are provided in Table I. It should be

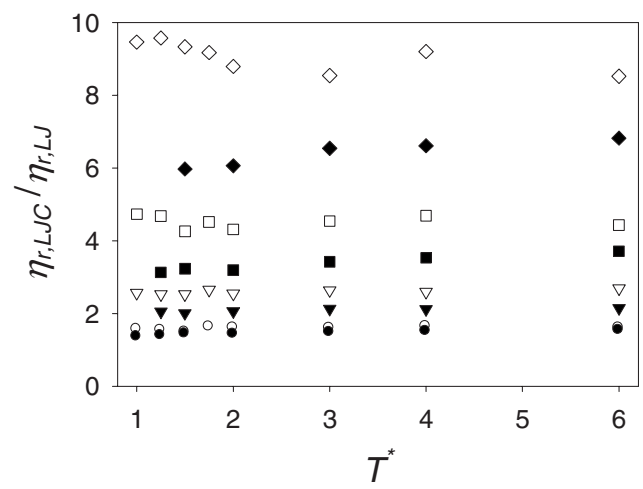

FIG. 7. LJC residual viscosity (relatively to the LJ one) versus temperature for different density $\left(\rho^{*}=0.8\right.$, solid symbols, and $\rho^{*}$ $=1$, open symbols) and different chain lengths: $\bigcirc, N=2 ; \nabla, N=4$; $\square, N=8$; and $\diamond, N=16$. 
TABLE I. Parameters values in Eqs. (12) and (13); the $b_{i}$ parameters come from Ref. [18].

\begin{tabular}{cccccccc}
\hline \hline$b_{1}$ & $b_{2}$ & $b_{3}$ & $b_{4}$ & $b_{5}$ & $b_{6}$ & $c_{1}$ & $c_{2}$ \\
\hline 0.062692 & 4.095577 & $-8.743269 \times 10^{-6}$ & 11.12492 & $2.542477 \times 10^{-6}$ & 14.863984 & 1.42 & 1.965 \\
\hline \hline
\end{tabular}

noted that Eq. (12) ensures that $\eta_{r, L J C}^{*}(N=1)=\eta_{r, L J}^{*}$.

\section{LJC Newtonian viscosity correlation}

In a previous work [18], we have developed an empirical correlation, based on extensive NEMD [18] and equilibrium MD [44] results, in order to estimate precisely the LJ residual viscosity:

$$
\eta_{r, L J}^{*}=b_{1}\left(e^{b_{2} \rho^{*}}-1\right)+b_{3}\left(e^{b_{4} \rho^{*}}-1\right)+\frac{b_{5}}{\left(T^{*}\right)^{2}}\left(e^{b_{6} \rho^{*}}-1\right),
$$

where the $b_{i}$ are numerical parameters adjusted on MD simulation data on the LJ fluid $(N=1)$, which are provided in Table I. This correlation, combined with the one for the zerodensity viscosity, Eq. (7), holds for $\rho^{*} \leqslant 1.275$ and $0.6 \leqslant T^{*}$ $\leqslant 6$ and yields, relatively to the MD data (338 points), an $\mathrm{AAD}=1.93 \%$ with a $\mathrm{MxD}=5.75 \%$ [18].

Thus, using these results, it is possible to obtain an estimation of $\eta_{r, L J C}^{*}$ by combining Eqs. (12) and (13). Then, by employing also Eq. (10), we get a relation that allows an estimation of the Newtonian viscosity of the LJC fluid (for $N \leqslant 16)$ :

$$
\begin{aligned}
\eta^{*}= & \frac{5}{16 \Omega_{v}} \sqrt{\frac{T^{*}}{N \pi}}+\left[b_{1}\left(e^{b_{2} \rho^{*}}-1\right)+b_{3}\left(e^{b_{4} \rho^{*}}-1\right)\right. \\
& \left.+\frac{b_{5}}{\left(T^{*}\right)^{2}}\left(e^{b_{6} \rho^{*}}-1\right)\right]\left[1+(N-1) \frac{\left(\rho^{*}\right)^{c_{1}}}{c_{2}}\right]
\end{aligned}
$$

where $b_{i}$ and $c_{i}$ are provided in Table I. Deviations between results yielded by the proposed correlation, Eq. (14), and all NEMD simulation data are given in Table II. Figure 8 illustrates the results of the proposed correlation, Eq. (14).

The results provided in Table II clearly show that the proposed correlation, Eq. (14), is able to guarantee a very good estimation of the Newtonian viscosity of the LJC fluid compared to NEMD results (taking into account uncertainties on the NEMD data, see the Appendix). A remarkable aspect of the correlation, Eq. (14), is that the quality of the predictions only slightly deteriorates when the chain length increases. This result is interesting to note if one considers the increasing uncertainties on $\eta^{*}$ with $N$ of the NEMD data. In addition (see Fig. 8), it is worth noting that the deviations between NEMD data and Eq. (14) results are rather well distributed along $T^{*}$ and $\rho^{*}$. Thus, we can consider that Eq. (14) adequately represents the LJC Newtonian viscosity for $N \leqslant 16$ over the domain $0 \leqslant \rho^{*} \leqslant 1.1$ and $0.7 \leqslant T^{*} \leqslant 6$ (except close to the critical point).

Finally, as the phase diagrams of the LJC, in dimensionless variables, evolve with $N$ [47] and so, as an example, the triple and critical point location varies with the chain length, it should be mentioned that some care should be taken when using the proposed correlation for applications to real fluids in order to keep a physical meaning of the results.

\section{E. Application to $n$-butane}

In order to show the reader the improvement induced by the LJC correlation over the LJ one for the estimation of the viscosity of a nonspherical molecule, we have used $n$-butane as an example. For estimating the number of segments of the LJC fluid model, we have employed a relation widely utilized to model $n$-alkane thermodynamic properties in the SAFT literature [48]:

$$
N=1+\frac{n_{c}-1}{3}
$$

where $n_{C}$ is the number of carbon atoms of normal alkane, which leads to $N=2$ for $n$-butane. Concerning the energy parameter $\varepsilon$, it has been deduced from the critical temperature for both LJ and LJC fluids-i.e., using

$$
\varepsilon=\frac{k_{B} T_{c}}{T_{c}^{*}},
$$

where $T_{c}=425.13 \mathrm{~K}$ for $n$-butane [40]; for the critical of the fluid model, we have employed $T_{c}^{*}=1.313$ for the $\mathrm{LJ}$ fluid $(N=1)$ [49] and $T_{c}{ }^{*}=1.784$ for the LJC fluid $(N=2)$ [50].

In order to perform an adjustment of $\sigma$ for both $N=1$ and $N=2$, we have used the viscosity database of Vogel et al. [51] on $n$-butane viscosity for $T=200,300,400$, and $500 \mathrm{~K}$
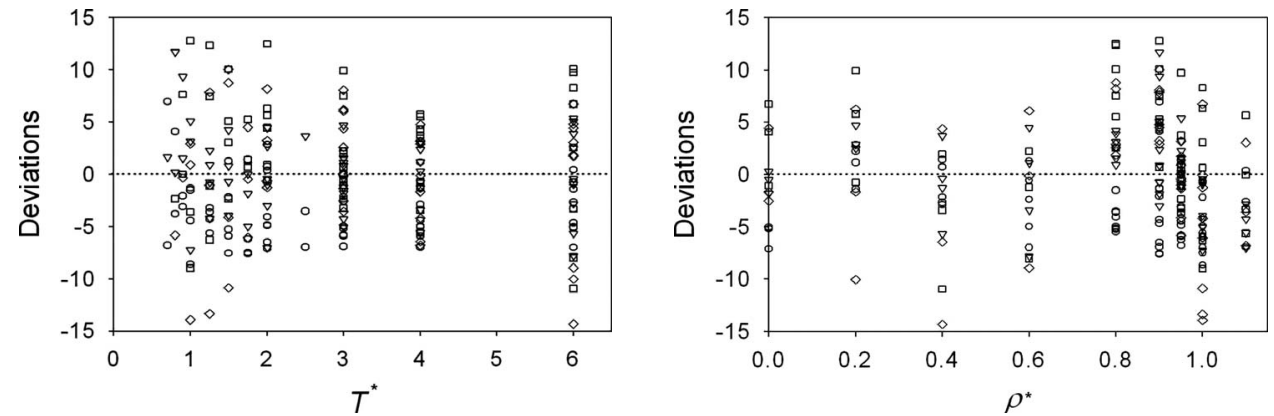

FIG. 8. Deviations (in percentage) between NEMD viscosity results and those provided by Eq. (14) for the LJC fluid: $\bigcirc, N=2$; $\nabla, N=4 ; \square, N=8$; and $\diamond, N=16$. 
TABLE II. Deviations between MD viscosity results and those provided by Eq. (14).

\begin{tabular}{lccccc}
\hline \hline & $N=1$ & $N=2$ & $N=4$ & $N=8$ & $N=16$ \\
\hline $\operatorname{AAD}(\%)$ & 1.93 & 4.16 & 3.08 & 4.94 & 4.93 \\
$\operatorname{MxD}(\%)$ & 5.25 & 8.62 & 11.69 & 12.79 & 14.32 \\
\hline \hline
\end{tabular}

and $P=0.1,25$, and $50 \mathrm{MPa}$. As the inputs of the LJ and LJC correlations are $T$ and $\rho$, the equation of state of Buecker and Wagner [52] has been used to deduce the density for the chosen states. It should be noted that, for this range of thermodynamic conditions, covering gas, liquid, and supercritical states, the shear viscosity vary from 7.45 to $702.53 \mu \mathrm{Pas}$. The adjustment has been done so that the AAD is minimized. The molecular parameters obtained are provided in Table III.

Using the molecular parameters provided in Table III, the LJ correlation yields an AAD equal to $10.3 \%(\mathrm{MxD}$ $=18.1 \%$ ) and the LJC correlation yields an $\mathrm{AAD}=3.5 \%$ $(\mathrm{MxD}=10.1 \%)$ relatively to the viscosity data of Vogel et al. [51]. This result is very interesting as it shows unambiguously that, using only $\sigma$ as adjustable parameter, the LJC fluid model outperforms the simple LJ one for an estimation of the $n$-butane viscosity. In addition, the rather low AAD obtained indicates that the LJC fluid provides a good modeling of the viscosity of $n$-butane, considering the wide range of thermodynamic conditions considered and the fact that only one parameter has been adjusted.

\section{CONCLUSIONS}

In this work, extensive nonequilibrium molecular dynamics simulations have been performed in order to estimate the Newtonian shear viscosity of the Lennard-Jones chain fluid for short chains $(N=1,2,4,8$, and 16) and a large range of thermodynamic conditions.

In a preliminary results part, a brief analysis of the influence of the shear rate and of the spring (bonded interactions) stiffness has been provided. As expected, NEMD results have shown that shear thinning appears only for the longest chains and for the largest shear rate. More precisely, it has been noted that the plateau-i.e., the Newtonian regime-is reached for shear-rate value that decreases when the chain length increases. Concerning the influence of the spring stiffness between segments, it has been noticed that $\eta^{*}$ becomes independent of $k^{*}$ when $k^{*}$ is above $\sim 1000$. Using these results, we have chosen the shear rate and the spring stiffness

TABLE III. Molecular parameters obtained for $n$-butane. $\varepsilon$ has been deduced from Eq. (16) and $N$ of the LJC fluid from Eq. (15); $\sigma$ has been adjusted on the viscosity data of Vogel et al. [51].

\begin{tabular}{cccccc}
\hline \hline & \multicolumn{2}{c}{ LJ fluid } & \multicolumn{3}{c}{ LJC fluid } \\
\hline$N$ & $\sigma(\mathrm{nm})$ & $\varepsilon(\mathrm{J} / \mathrm{mol})$ & $N$ & $\sigma(\mathrm{nm})$ & $\varepsilon(\mathrm{J} / \mathrm{mol})$ \\
\hline 1 & 0.5036 & 2692.11 & 2 & 0.4 & 1981.36 \\
\hline \hline
\end{tabular}

so that the systematic evaluation of the LJC shear viscosity is consistent; i.e., the regime remains Newtonian and the fluids studied are effectively composed of jointed chains with a bond length of $\sigma$.

In a second part, we have proposed an approach to estimate the zero-density shear viscosity $\eta_{0}^{*}$ using NEMD simulations at nonzero density. To do so, using the microscopic formulation of the linear momentum flux, we have shown that, for the LJ fluid $(N=1)$, the translational viscosity computed using NEMD simulations at $\rho^{*}=0.2$ is very close to the zero-density viscosity. Using this approach, we have estimated the zero-density viscosity of the LJC fluid for $N=2$, 4, 8, and 16. Employing these results, we have deduced that, for a given temperature, $\eta_{0}^{*}$ of a LJC fluid is very well approximated by $\eta_{0}^{*}$ of the LJ fluid divided by $N^{1 / 2}$. It should be noted that this result is supported by a rough analysis of the behavior of the zero-density viscosity of the hard-sphere chain fluid.

Then, based on NEMD results for a wide range of thermodynamic conditions, covering gas, liquid, and supercritical conditions, $\rho^{*}$ varying from 0.2 and 1.1 and $T^{*}$ varying from 0.7 to 6 , we have proposed an empirical chain term (consistent with the Rouse model, i.e., a linear increase of the residual viscosity $\eta_{r}{ }^{*}$, with $N$ ) which describes very well the residual viscosity of the LJC fluid relatively to that of the LJ fluid. Next, using these results, is proposed a complete correlation composed of both the zero-density and the residual contributions to the shear viscosity and combined with a LJ reference term. It is shown that this empirical correlation is able to provide an excellent estimation of the LJC fluid viscosity for $N \leqslant 16$ over the domain $\rho^{*} \leqslant 1.1$ and 0.7 $\leqslant T^{*} \leqslant 6$, with deviations always below $14.4 \%$ compared to NEMD results. In addition, it is shown that the quality of the predictions of the proposed correlation only slightly deteriorates when the chain length increases, the AAD deviations with MD simulations being always smaller than $5 \%$ whatever $N$.

Finally, the LJ and LJC viscosity correlations are applied in order to estimate the shear viscosity of $n$-butane for a temperature varying from 200 to $500 \mathrm{~K}$ and a pressure varying from 0.1 to $50 \mathrm{MPa}$. For both models, only one molecular parameter, the segment diameter, is adjusted. It has been obtained that the LJ correlation yields an $\mathrm{AAD}=10.3 \%$ $(\mathrm{MxD}=18.1 \%)$ and the LJC correlation yields an AAD $=3.5 \%(\mathrm{MxD}=10.1 \%)$. This result shows unambiguously that, using only $\sigma$ as adjustable parameter, the LJC model outperforms the simple LJ for such a molecule and is able to provide a very reasonable estimation of the shear viscosity of $n$-butane for a wide range of thermodynamic conditions.

\section{ACKNOWLEDGMENTS}

We gratefully acknowledge computational facilities provided by the UPPA and the TREFLE laboratory, the supercomputer of which has been financially supported by the Conseil Régional d'Aquitaine.

\section{APPENDIX}

Results of simulations for a large range of stable thermodynamic conditions are given in Table IV. 
TABLE IV. Shear viscosity of the LJC fluid computed by NEMD simulations.

\begin{tabular}{|c|c|c|c|c|c|}
\hline$\rho^{*}$ & $T^{*}$ & $N=2$ & $N=4$ & $N=8$ & $N=16$ \\
\hline 0.2 & 2 & $0.227 \pm 0.012$ & & & \\
\hline 0.2 & 3 & $0.285 \pm 0.015$ & $0.228 \pm 0.010$ & $0.193 \pm 0.015$ & $0.202 \pm 0.015$ \\
\hline 0.2 & 4 & $0.337 \pm 0.022$ & $0.266 \pm 0.014$ & $0.224 \pm 0.014$ & $0.236 \pm 0.02$ \\
\hline 0.2 & 6 & $0.431 \pm 0.038$ & $0.325 \pm 0.021$ & $0.281 \pm 0.025$ & $0.291 \pm 0.03$ \\
\hline 0.4 & 1.75 & $0.423 \pm 0.08$ & & & \\
\hline 0.4 & 2 & $0.442 \pm 0.09$ & & & \\
\hline 0.4 & 2.5 & $0.475 \pm 0.013$ & $0.477 \pm 0.013$ & & \\
\hline 0.4 & 3 & $0.514 \pm 0.012$ & $0.516 \pm 0.015$ & $0.603 \pm 0.01$ & $0.835 \pm 0.025$ \\
\hline 0.4 & 4 & $0.569 \pm 0.013$ & $0.556 \pm 0.014$ & $0.662 \pm 0.016$ & $0.95 \pm 0.04$ \\
\hline 0.4 & 6 & $0.654 \pm 0.021$ & $0.645 \pm 0.027$ & $0.765 \pm 0.024$ & $1.072 \pm 0.052$ \\
\hline 0.6 & 1.5 & $0.940 \pm 0.035$ & & & \\
\hline 0.6 & 2 & $0.987 \pm 0.046$ & $1.212 \pm 0.047$ & & \\
\hline 0.6 & 3 & $1.061 \pm 0.038$ & $1.288 \pm 0.032$ & $1.872 \pm 0.083$ & $3.01 \pm 0.19$ \\
\hline 0.6 & 4 & $1.141 \pm 0.025$ & $1.383 \pm 0.056$ & $1.960 \pm 0.068$ & $3.21 \pm 0.2$ \\
\hline 0.6 & 6 & $1.255 \pm 0.054$ & $1.512 \pm 0.061$ & $2.15 \pm 0.09$ & $3.55 \pm 0.24$ \\
\hline 0.8 & 1 & $2.73 \pm 0.1$ & & & \\
\hline 0.8 & 1.25 & $2.62 \pm 0.15$ & $3.78 \pm 0.23$ & $5.71 \pm 0.52$ & \\
\hline 0.8 & 1.5 & $2.58 \pm 0.12$ & $3.52 \pm 0.22$ & $5.60 \pm 0.4$ & $10.3 \pm 1.4$ \\
\hline 0.8 & 2 & $2.48 \pm 0.1$ & $3.45 \pm 0.22$ & $5.26 \pm 0.35$ & $9.91 \pm 1.31$ \\
\hline 0.8 & 2.5 & $2.45 \pm 0.06$ & & & \\
\hline 0.8 & 3 & $2.49 \pm 0.12$ & $3.42 \pm 0.16$ & $5.36 \pm 0.37$ & $10.1 \pm 0.7$ \\
\hline 0.8 & 4 & $2.53 \pm 0.12$ & $3.37 \pm 0.24$ & $5.43 \pm 0.32$ & $10.0 \pm 1.3$ \\
\hline 0.8 & 6 & $2.59 \pm 0.08$ & $3.37 \pm 0.16$ & $5.58 \pm 0.32$ & $10.1 \pm 0.9$ \\
\hline 0.9 & 0.7 & $7.57 \pm 0.66$ & & & \\
\hline 0.9 & 0.8 & $6.70 \pm 0.69$ & $10.0 \pm 1.1$ & & \\
\hline 0.9 & 0.9 & $6.34 \pm 0.49$ & $9.08 \pm 0.61$ & $16.2 \pm 2.4$ & \\
\hline 0.9 & 1 & $5.74 \pm 0.39$ & $8.61 \pm 0.8$ & $14.0 \pm 2.0$ & $28.6 \pm 6.8$ \\
\hline 0.9 & 1.25 & $5.05 \pm 0.34$ & $7.75 \pm 0.87$ & $12.5 \pm 1.6$ & $23.2 \pm 3$ \\
\hline 0.9 & 1.5 & $4.75 \pm 0.28$ & $7.01 \pm 0.62$ & $11.6 \pm 1.3$ & $20.5 \pm 3.8$ \\
\hline 0.9 & 1.75 & $4.47 \pm 0.3$ & $6.65 \pm 0.38$ & $10.8 \pm 0.9$ & $20.2 \pm 2.4$ \\
\hline 0.9 & 2 & $4.24 \pm 0.32$ & $6.44 \pm 0.44$ & $10.4 \pm 1.2$ & $19.5 \pm 2.3$ \\
\hline 0.9 & 3 & $3.97 \pm 0.15$ & $5.71 \pm 0.34$ & $9.90 \pm 1.4$ & $17.1 \pm 2.1$ \\
\hline 0.9 & 4 & $3.68 \pm 0.15$ & $5.48 \pm 0.44$ & $9.27 \pm 0.9$ & $17.0 \pm 2.6$ \\
\hline 0.9 & 6 & $3.81 \pm 0.22$ & $5.27 \pm 0.4$ & $8.61 \pm 0.8$ & $16.7 \pm 1.7$ \\
\hline 0.95 & 0.7 & $15.4 \pm 1.9$ & $23.2 \pm 0.28$ & & \\
\hline 0.95 & 0.8 & $12.4 \pm 1.4$ & $19.5 \pm 2.0$ & $35.6 \pm 3.8$ & $69.3 \pm 13.5$ \\
\hline 0.95 & 0.9 & $10.6 \pm 0.9$ & $16.6 \pm 2.3$ & $29.9 \pm 4.6$ & $56.3 \pm 11.8$ \\
\hline 0.95 & 1 & $9.5 \pm 1.0$ & $14.4 \pm 1.7$ & $27.4 \pm 3.4$ & $49.1 \pm 8$ \\
\hline 0.95 & 1.25 & $7.5 \pm 0.5$ & $11.6 \pm 1.2$ & $21.3 \pm 3.0$ & $39.9 \pm 7.5$ \\
\hline 0.95 & 1.5 & $6.7 \pm 0.5$ & $10.2 \pm 0.9$ & $18.6 \pm 2.3$ & $35.5 \pm 5.1$ \\
\hline 0.95 & 1.75 & $6.1 \pm 0.4$ & $9.27 \pm 0.74$ & $16.2 \pm 1.5$ & $30.9 \pm 4.9$ \\
\hline 0.95 & 2 & $5.62 \pm 0.36$ & $8.73 \pm 0.60$ & $15.2 \pm 2.1$ & $28.8 \pm 4.6$ \\
\hline 0.95 & 3 & $4.99 \pm 0.35$ & $7.62 \pm 0.75$ & $13.7 \pm 1.8$ & $25.1 \pm 4.5$ \\
\hline 0.95 & 4 & $4.71 \pm 0.29$ & $7.07 \pm 0.69$ & $12.1 \pm 1.2$ & $22.7 \pm 4.2$ \\
\hline 0.95 & 6 & $4.39 \pm 0.33$ & $6.57 \pm 0.61$ & $11.0 \pm 1.2$ & $22.1 \pm 3$ \\
\hline 1 & 0.8 & $29.9 \pm 0.48$ & $47.7 \pm 12.5$ & $83.6 \pm 19.0$ & \\
\hline 1 & 0.9 & $22.0 \pm 3.1$ & $35.5 \pm 5.2$ & $62.6 \pm 12.0$ & $122 \pm 42$ \\
\hline 1 & 1 & $17.2 \pm 1.4$ & $28.3 \pm 3.5$ & $52 \pm 7.8$ & $104 \pm 26$ \\
\hline 1 & 1.25 & $12.5 \pm 0.9$ & $20.5 \pm 4.5$ & $37.8 \pm 6.4$ & $77.3 \pm 18.6$ \\
\hline 1 & 1.5 & $9.89 \pm 0.81$ & $16.8 \pm 1.8$ & $28.1 \pm 2.8$ & $61.4 \pm 13.4$ \\
\hline 1 & 1.75 & $9.08 \pm 0.74$ & $14.7 \pm 1.7$ & $24.9 \pm 1.8$ & $50.4 \pm 11.4$ \\
\hline 1 & 2 & $7.97 \pm 0.39$ & $12.6 \pm 1.6$ & $21.2 \pm 1.94$ & $43.1 \pm 7.6$ \\
\hline 1 & 2.5 & $7.12 \pm 0.47$ & & & \\
\hline 1 & 3 & $6.50 \pm 0.41$ & $10.6 \pm 1.1$ & $18.1 \pm 2.6$ & $33.9 \pm 5.9$ \\
\hline 1 & 4 & $5.99 \pm 0.32$ & $9.26 \pm 0.85$ & $16.5 \pm 2.6$ & $32.2 \pm 6.2$ \\
\hline 1 & 6 & $5.38 \pm 0.32$ & $8.69 \pm 0.74$ & $14.1 \pm 1.7$ & $26.9 \pm 5.9$ \\
\hline 1.1 & 2 & $18.8 \pm 2.3$ & $35.1 \pm 6.1$ & 56.9 & \\
\hline 1.1 & 3 & $12.2 \pm 1.1$ & $21.3 \pm 2.2$ & $37.6 \pm 4.4$ & $74.5 \pm 17.5$ \\
\hline 1.1 & 4 & $10.2 \pm 0.9$ & $17.1 \pm 2.4$ & $31.4 \pm 4.1$ & $60.8 \pm 17.9$ \\
\hline 1.1 & 6 & $8.04 \pm 0.8$ & $13.5 \pm 0.9$ & $24.9 \pm 2.8$ & $44.5 \pm 7.9$ \\
\hline
\end{tabular}


[1] S. Chapman and T. G. Cowling, The Mathematical Theory of Non-Uniform Gases Cambridge Mathematical Library (Cambridge University Press, Cambridge, England, 1970).

[2] J. P. Hansen and I. R. MacDonald, Theory of Simple Liquids (Academic Press, New York, 1990).

[3] M. P. Allen and D. J. Tildesley, Computer Simulations of Liquids, Oxford Science Publications (Oxford University Press, Oxford, 1987).

[4] I. Nezbeda, Fluid Phase Equilib. 182, 3 (2001).

[5] E. Müller and K. Gubbins, Ind. Eng. Chem. Res. 40, 2193 (2001).

[6] H. Sigurgeirsson and D. M. Heyes, Mol. Phys. 101, 469 (2003).

[7] D. D. Royal, V. Vesovic, J. P. M. Trusler, and W. A. Wakeham, Mol. Phys. 101, 339 (2003).

[8] A. S. De Wijn, V. Vesovic, G. Jackson, and J. P. M. Trusler, J. Chem. Phys. 128, 204901 (2008).

[9] R. Srivastava, D. K. Dwivedee, and K. N. Khanna, J. Mol. Liq. 139, 29 (2008)

[10] K. Foteinopoulou, N. K. Karayiannis, M. Laso, M. Kröger, and M. Mansfield, Phys. Rev. Lett. 101, 265702 (2008).

[11] R. Laghaei, A. E. Nasrabad, and B. C. Eu, J. Chem. Phys. 123, 234507 (2005).

[12] M. Robles and L. I. Uruchurtu, J. Chem. Phys. 124, 094112(2006).

[13] L. V. Woodcock, AIChE J. 52, 438 (2006).

[14] S. A. Egorov, J. Chem. Phys. 128, 144508 (2008).

[15] R. Khordad, Physica A 387, 4519 (2008).

[16] R. L. Rowley and M. M. Painter, Int. J. Thermophys. 18, 1109 (1997).

[17] M. S. Zabaloy, J. M. V. Machado, and E. A. Macedo, Int. J. Thermophys. 22, 829 (2001).

[18] G. Galliero, C. Boned, and A. Baylaucq, Ind. Eng. Chem. Res. 44, 6963 (2005).

[19] M. S. Zabaloy, J. M. V. Machado, and E. A. Macedo, J. Supercrit. Fluids 36, 106 (2005).

[20] C. K. Zeberg-Mikkelsen, G. Watson, A. Baylaucq, G. Galliero, and C. Boned, Fluid Phase Equilib. 245, 6 (2006).

[21] G. Galliero and C. Boned, Fluid Phase Equilib. 269, 19 (2008).

[22] E. Ruckenstein and H. Liu, Ind. Eng. Chem. Res. 36, 3927 (1997).

[23] G. Galliero, C. Boned, A. Baylaucq, and F. Montel, Phys. Rev. E 73, 061201 (2006).

[24] F. Müller-Plathe, Phys. Rev. E 59, 4894 (1999).

[25] J. K. Johnson, E. A. Müller, and K. E. Gubbins, J. Phys. Chem. 98, 6413 (1994).

[26] Y.-X. Yu and G.-H. Gao, Fluid Phase Equilib. 166, 111 (1999).
[27] R. A. Reis, F. C. Silva, R. Nobrega, J. V. Oliveira, and F. W. Tavares, Fluid Phase Equilib. 221, 25 (2004).

[28] O. Suarez-Iglesias, I. Medina, C. Pizarro, and J. L. Bueno, Chem. Eng. Sci. 62, 6499 (2007).

[29] M. Kröger, Phys. Rep. 390, 453 (2004).

[30] O. Peleg, M. Kröger, I. Hecht, and Y. Rabin, Europhys. Lett. 77, 58007 (2007).

[31] S. Hess, J. Non-Newtonian Fluid Mech. 23, 305 (1987).

[32] P. Bordat and F. Müller-Plathe, J. Chem. Phys. 116, 3362 (2002).

[33] G. Galliero and C. Boned, J. Chem. Phys. 129, 074506 (2008).

[34] J. M. Haile, Molecular Dynamics Simulation: Elementary methods (Wiley, New York, 1992).

[35] H. J. C. Berendsen, J. P. M. Postma, W. F. van Gunsteren, A. di Nola, and J. R. Haak, J. Chem. Phys. 81, 3684 (1984).

[36] T. J. Müller, M. Al-Samman, and F. Müller-Plathe, J. Chem. Phys. 129, 014102(2008)

[37] D. M. Heyes, J. Chem. Phys. 85, 997 (1986).

[38] S. Hess, Int. J. Thermophys. 23, 905 (2002).

[39] J. Millat, J. H. Dymond, and C. A. Nieto de Castro, Transport Properties of Fluids: Their Correlation, Prediction and Estimation (Cambridge University Press, Cambridge, England, 1996).

[40] B. E. Poling, J. M. Prausnitz, and J. P. O'Connell, The Properties of Gases and Liquids (McGraw-Hill, New York, 2001).

[41] P. D. Neufeld, A. R. Janzen, and R. A. Aziz, J. Chem. Phys. 57, 1100 (1972).

[42] G. Galliero, C. Nieto-Draghi, C. Boned, J. B. Avalos, A. D. Mackie, A. Baylaucq, and F. Montel, Ind. Eng. Chem. Res. 46, 5238 (2007).

[43] M. Bugel and G. Galliero, Chem. Phys. 352, 249 (2008).

[44] K. Meier, A. Laesecke, and S. Kabelac, J. Chem. Phys. 121 3671 (2004).

[45] M. Doi and S. F. Edwards, The theory of Polymer Dynamics (Oxford University Press, Oxford, 1986).

[46] M. Kröger and S. Hess, Phys. Rev. Lett. 85, 1128 (2000).

[47] F. J. Blas, A. Galindo, and C. Vega, Mol. Phys. 101, 449 (2003).

[48] T. Lafitte, D. Bessieres, M. M. Piñeiro, and J. L. Daridon, J. Chem. Phys. 124, 024509 (2006).

[49] H. Okumura and F. Yonezawa, J. Chem. Phys. 113, 9162 (2000).

[50] F. A. Escobedo and J. J. De Pablo, Mol. Phys. 87, 347 (1996).

[51] E. Vogel, C. Küechenmeister, and E. Bich, High Temp. - High Press. 31, 173 (1999).

[52] D. Buecker and W. Wagner, J. Phys. Chem. Ref. Data 35, 929 (2006). 